\title{
I normanni nel territorio dell'Albania del Sud. Il caso di Gurëzeza
}

Suela Xhyheri, Skënder Muçaj e Skënder Bushi

\section{Q OpenEdition \\ 1 Journals}

Edizione digitale

URL: https://journals.openedition.org/cem/15820

DOI: $10.4000 /$ cem. 15820

ISSN: 1954-3093

Editore

Centre d'études médiévales Saint-Germain d'Auxerre

Notizia bibliografica digitale

Suela Xhyheri, Skënder Muçaj et Skënder Bushi, «I normanni nel territorio dell'Albania del Sud. II caso di Gurëzeza », Bulletin du centre d'études médiévales d'Auxerre | BUCEMA [En ligne], 22.2 | 2018, mis en ligne le 23 janvier 2019, consulté le 22 septembre 2022. URL : http://journals.openedition.org/cem/ 15820 ; DOI : https://doi.org/10.4000/cem.15820

Questo documento è stato generato automaticamente il 22 septembre 2022.

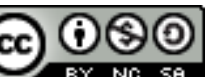

Creative Commons - Attribution - Pas d'Utilisation Commerciale - Partage dans les Mêmes Conditions 4.0 International - CC BY-NC-SA 4.0

https://creativecommons.org/licenses/by-nc-sa/4.0/ 


\section{I normanni nel territorio} dell'Albania del Sud. Il caso di

Gurëzeza

Suela Xhyheri, Skënder Muçaj e Skënder Bushi

La presenza normanna in Albania 
1 Dopo la conquista di Bari nel 1071, Roberto Guiscardo è riuscito a unificare tutti i territori dei principi normanni del Sud Italia, facendo nascere un regno potente, che avanzò le sue pretese territoriali verso l'altra sponda dell'Adriatico. Questa nuova dinamica nei rapporti Est - Ovest, ha posto il territorio albanese nel centro di un teatro di guerra. L'Albania rappresentava il confine occidentale di Bisanzio, mentre per la nuova potenza dell'Ovest Europa, era la strada verso la conquista di Costantinopoli. Di conseguenza, il territorio dell'odierna Albania si trasformò da lì a poco in un luogo di mille battaglie, non solo militari ma bensì culturali e religiose ${ }^{1}$.

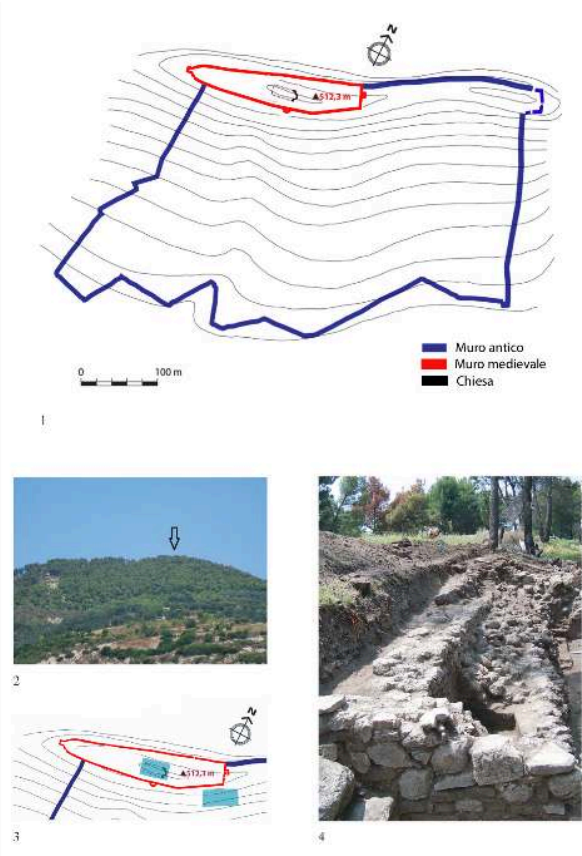

2 Nella primavera del 1081, un grande esercito normanno partì dal porto di Brindisi e Otranto, sotto la guida di Guiscardo e di suo figlio Boemundo ${ }^{2}$. Quest'ultimo con parte della flotta sbarcò sulla costa di Valona, riuscendo, poi, a conquistare velocemente non solo la città stessa, ma anche dei luoghi strategici come Kanina, Jeriko (Orikum), Glavinica ${ }^{3}$ ecc. Il vero obbiettivo era la conquista della città principale sull'Adriatico, Durrazzo. Gli sforzi per la sua difesa risultarono inutili, e la città cade sotto i normanni ${ }^{4}$.

3 Dopo la conquista di Durrazzo, i normanni continuarono verso est, riuscendo a mettere sotto controllo l'ultimo territorio del «corridoio» Valona - Prespa, quello di Devoll 5 . Nella primavera del 1082 Robert Guiscardo, fu costretto a fare ritorno in Italia, e suo figlio Boemundo, non riuscì a controllare a lungo i territori conquistati. Dopo la morte di Guiscardo (1085), i normanni si ritirarono dall'odierna Albania, per fare ritorno nel 1107, guidati da Boemundo ${ }^{6}$. La loro flotta navale sbarcò nelle vicinanze di Valona nell'ottobre di quell'anno, per proseguire poi, via terra, verso Durrazzo ${ }^{7}$. Questa volta la città riuscì ad organizzare una difesa più serrata, facendo fronte agli attacchi dei normanni. Senza rinunciare all'assedio della città Boemundo continuò la sua marcia verso est. L'imperatore Alessio Comneno provò in tutte le maniere a fermare l'avanzamento dei normanni, bloccando i passaggi verso Ohrid e Dibra (Dober). Lui stesso era posizionato nella zona di Ohrid, Devoll e Kolonja, da dove comandava le sue truppe impegnate nei combattimenti nella regione di Arbër, ed a Valona e Glavinica ${ }^{8}$. Dopo mesi di combattimenti, l'esercito normanno bloccato nella Pianura Occidentale, $\mathrm{fu}$ costretto a rinunciare e fare rientro in Italia. A fine settembre del 1108, Boemundo si arrese, nel campo dell'Imperatore bizantino Deabolis.

4 La presenza dei normanni in Glavinica è testimoniato dall'iscrizione sulla tomba rinvenuta a Ballsh, dedicata al cavaliere Roberto di Monteforte ${ }^{9}$, che è morto e sepolto a Ballsh, come sottoufficiale dei normanni e degli inglesi in una crociata. Il cavaliere Roberto, molto probabilmente è la stessa persona che, riferendosi a Historia Ecclesiae di Odericus Vitalis nel 1107, fu espulso dall'Inghilterra andando ad Apulia da Boemondo di Taranto $^{10}$. Partecipò all'assedio di Durrazzo e morì in Albania nel $1108^{11}$. Essendo la sua 
figura rimasta fuori dalle attenzioni degli storici, ci siamo preposti a riportare una breve ricerca riguardante la sua genealogia.

Per conoscere la vera storia di Roberto di Montfort, dobbiamo partire dal dato più certo che disponiamo di lui, il cognome, della nobile famiglia Montfort (fr.). In Francia esistono due nobili famiglie con lo stesso cognome con origini a partire dal XI-XII secolo, una di Monfort-l'Amaury (provincia di Rambouie), dove nel 996 il Re Roberto II Capeto (971-1031) costruì un dongione, l'altra a Monfort-sur-Risle nella Normandia di sopra con un castello di proprietà costruito nel XI secolo. Dalle ricerche fatte dai vari studiosi sulla genealogia di queste famiglie, risulta che Roberto di Monteforte, che viene nominato a Ballsh, appartiene alla famiglia di Monfort-sur-Risle ${ }^{12}$. Membro famoso della stessa famiglia è anche Hugo II di Monteforte (?-1083), che divenne famoso nella battaglia di Hastings in Inghilterra dove riuscì ad ottenere come ricompensa dagli inglesi 114 proprietà. Per Hugo II di Monteforte (secundus Hugo) non si sa molto ma, dopo la morte della sua prima moglie, si risposò (aliam) ottenendo da quest'ultimo matrimonio « Hugonem Tertium et Robertum fratrem eius». Secondo le Gesta Normannorum Ducum, Hugo III e Roberto erano fratelli da parte del padre Hugo II, specificando anche che entrambi morirono in pellegrinaggio senza avere dei figli ${ }^{13}$. «(...) Robert de Monfort (...) » era testimone di un atto di donazione senza data, nella quale Roberto II duca di Normandia, donava terreni a Santo Stefano di Caen ${ }^{14}$. Tutte le sue proprietà furono confiscate dopo che venne accusato di tradimento nel 1107. Lui era a Durrazzo nel 1108. Quando Enrico II, Re d'Inghilterra riconferma le proprietà dell'abazia di Bec, in quest'ultime venivano inclusi i doni di « (...) Robert de Mone Forti Adeline sororis ipsirus Roberti », secondo l'atto firmato nel 1181/1189 ${ }^{15}$.

6 Alla guida degli figli di Alessio I, Giovanni II (1118-1143) e Manuele I Comneno (1143-1180), i normanni ed i bizantini, cercarono di conquistare territori oltre l'Adriatico. Nel 1149 il Re Ruggero II di Sicilia, conquista l'isola di Corfù e le coste dell'Epirio, mentre Manuele I Comneno nel 1155, con una flotta bizantina salpata dalle coste albanesi, riesce a conquistare la costa da Ancona a Taranto. Molto velocemente però, le truppe bizantine, furono costrette ad abbandonare le loro conquiste territoriali, costrette dalla coalizione capeggiata dal Re Normanno Guglielmo II e dal Vaticano.

7 Nel 1185 Re Guglielmo II fece gli ultimi sforzi della dinastia normanna in Italia per occupare i territori bizantini. Inizialmente, assieme alle città ed ai castelli del territorio di Valona conquistarono anche Durrazzo, per arrivare tramite via Egnatia fino a Salonicco, che fu consegnata dalle truppe della guarnigione Kunavija. Questo tragitto dell'esercito tramite la via Egnazia è documentato dalle monete di Re Guglielmo II, rinvenute nella chiesa di Scampis ${ }^{16}$ (l'odierno Elbasan). Comunque, Durrazzo ed alcune isole del mare Ionio, rimasero per un po' di tempo sotto i normanni. Guglielmo II venne susseguito dal sovrano tedesco Enrico VI, che inaugurò il dominio della dinastia Hofenshtaufen nell'Italia del Sud. I nuovi sovrani, assieme al regno, ereditarono dai loro predecessori la politica delle conquiste verso Est. A conferma di quello espansionismo, secondo il cronista bizantino Niceta Coniata $^{17}$, Re Enrico VI Hohenshtaufen si considerava « signore delle terre da Durrazzo a Salonicco ». 


\section{I siti de Gurëzeza e Mashkjeza}

8 Oltre ai dati storici sulla presenza normanna in Albania ci sono anche quelli scarsi e frammentati, archeologici. Particolarmente discussi in questo articolo sono due siti adiacenti e contemporanei : Gurëzeza e Mashkjeza, situati nel sudovest dell'Albania vicino alla città antica di Apollonia (fig. 1).

Fig. 1 - Gurëzeza e altri centri antichi intorno ad essa

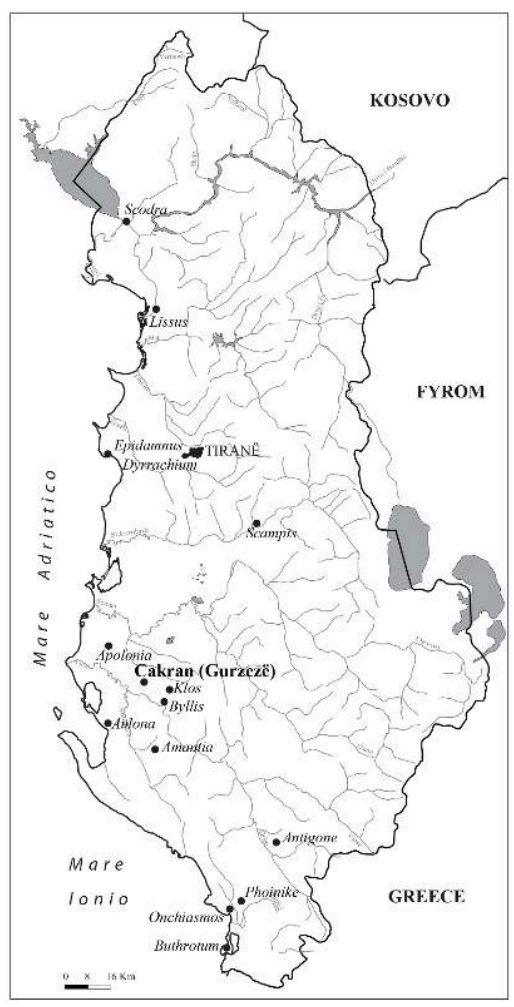

S. Bushi

9 Gli scavi sulla collina di Gurëzeza sono iniziati nel 1983 da Neritan Ceka e Skender Muçaj, nella quale sono stati individuati solo le rovine del periodo antico di questa città, che ha i suoi inizi nel V secolo avanti Cristo. Nel 2009 questi scavi sono ripresi scoprendo le strutture di una fortificazione, tra cui una torre, un edificio di culto, una residenza signorile, una cisterna e materiale ceramico.

Questo sito è anche conosciuto come Le due Pietre (al. Gurëzeza). Il castello di Mylus (al. Gurëzeza $\left.{ }^{18}\right)$ appartiene all'età medievale ed è costruita sopra le rovine della città antica di Gurëzeza (fig. 2). 
Fig. 2 - Gurëzeza. La fortificazione della città medievale

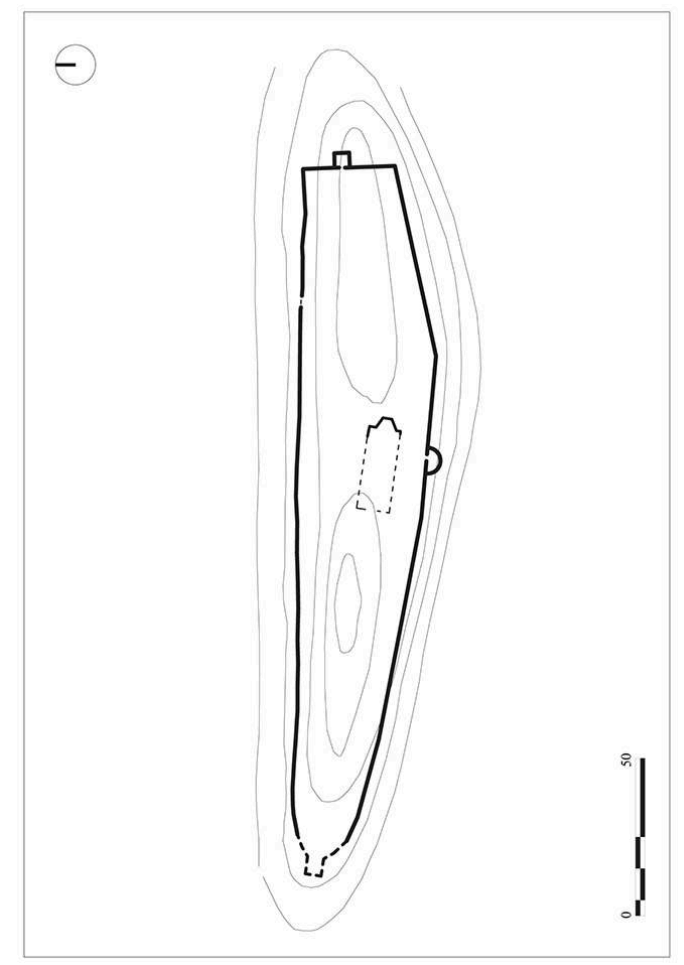

S. Bushi

11 Viene riconosciuta anche con i nomi : "le Pietre Nere ", "Mylys ", "le Due Pietre Nere ». La collina delle Pietre Nere si trova nella catena collinare che inizia da Apollonia e finisce in Gradishtë di Hekal. La collina si sviluppa su un dorso di 30-40 mt. verso nordovest - sud-est, avendo due sommità collegate tra di loro. Dalla sua altezza si controlla la pianura di Cakran e la valle del fiume Gjanica. Sul dorso della collina passava la via che collegava Apollonia con Bylis. Qafa e Shkallës ad est della collina garantiva un collegamento naturale con la valle di Vjosa e di Gjanica. Nel periodo ellenistico è stato utilizzato per il collegamento dei centri abitati della valle di Shushica con la città di Gurëzeza, Margëlliç e Dimal.

Il pendio sud-ovest della collina dove si estendeva la città antica, ha una pendenza di circa $30^{\circ}$, per trasformarsi in un terrazzamento fino a $100 \mathrm{mt}$. nella direzione fra le due creste. La parte della collina fra le due creste ed il pendio sud-ovest, un tempo erano recintati da una fortificazione ellenistica, mentre la cresta a nord-ovest è stata fortificata nel medioevo (fig.3/2). Nella parte più alta della collina delle Pietre Nere, dove nel periodo ellenistico fiorì una delle città byline ${ }^{19}$, durante il medioevo, si costruirono le mura di un castello (fig. 3/1). 
Fig. 3 - Gurëzeza. La Pianta della città medievale

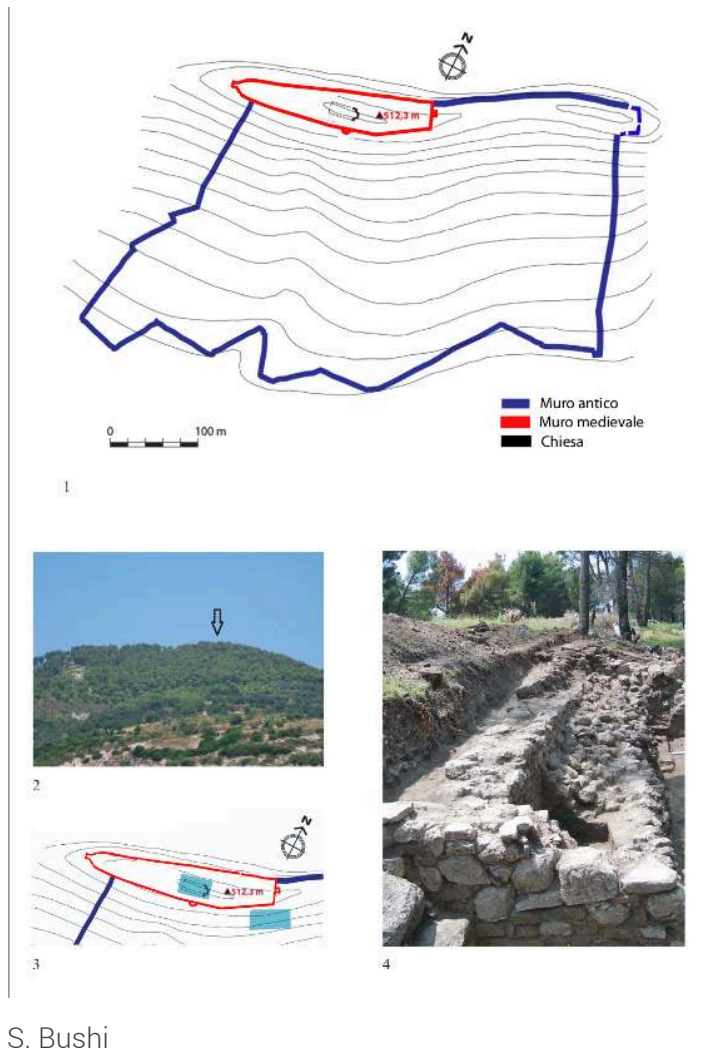

Per la prima volta il castello di Mylus è stato menzionato da Anna Comnena, inizialmente sotto Boemundo ${ }^{20}$, e poi nei tentativi di Cantacuzeno per riconquistarla ai franchi ${ }^{21}$. A partire da questi dati, di fatto, i vari autori lo hanno identificato in luoghi diversi, partendo da Durrazzo, Devoll o nella provincia di Mallakastra. Negli eventi del XIII-XIV secolo che hanno luogo in questi territori, si nomina un nome in modo sommario "Le montagne di Spinarica (al. Malet e Spinaricës)» ${ }^{22}$. Un dato che corrisponde al nome che porta oggi, la troviamo negli scritti di Giovanni Musachi quando parla del confine sud di Mallacastra fino al fiume Vjosa, nel luogo conosciuto come « Le Due Pietre ", nei registri ottomani del $1519-1520$ e 1570 viene nominato solo come Cakran.

La mancata nomina del castello durante le vicende del XIII-XIV secolo, nei territori dove viene nominato Malet e Spinaricës ${ }^{23}$, e la mancanza di un toponimo simile ha reso difficilissima la sua identificazione con uno dei castelli medievali della zona. Partendo dalla prima notizia di Anna Comnena che posiziona la fortezza di Mylus in relazione con Petrula, al di là del fiume Deabolis - il nome medievale del fiume Devoll -, si può ipotizzare la sua presenza fra il fiume Vjosa e Deabolis. Si tratta di estendere le ricerche in una vasta area fra Myzeqe, Berat e Mallakastër. Nella seconda notizia questo spazio si ristringe verso la riva destra del fiume Vjosa e molto vicino a Glavinica ${ }^{24}$, ma bisogna tenere in considerazione solo la parte navigabile del fiume Vjosa. Nella parte destra della valle di Vjosa sono riconosciute le rovine di Gradishtë di Klos, Gradishtë di Bylis, Gradishtë di Drenova, Gradishtë di Belishova, Qyteza (Mashkjeza), Gurëzeza, ed il Monastero di Apollonia ${ }^{25}$. In relazione con Glavinica, ma più lontano del fiume Vjosa, possiamo elencare le rovine di Gradishtë di Rabie, il Castello di Cfiri, il castello di Margëlliç̧̧ e la Gradishtë di Mbjeshova ${ }^{27}$. Questi dati non escludono totalmente la presenza di qualche 
altro castello, soprattutto nella pianura, le cui rovine potrebbero essere state coperte dai depositi del fiume Vjosa.

Anna Comnena ha definito Mylus come un piccolo castello, escludendo così Bylis ed il gruppo dei castelli vicino a Vjosa. Rimangono così soltanto tre castelli; Gurëzeza, Mashkjeza ed il Monastero di Apollonia. A. Ducellier, riferendosi al fatto che il generale bizantino prima di occupare Glaviniza attaccò Mylus per poter interrompere il collegamento fra Valona e Durrazzo e non permettere ai normanni di rinforzarsi con altre truppe via mare, propone Apollonia come la locazione più probabile ${ }^{28}$. Dagli scavi fatti nel Monastero di Apollonia, in vari ambienti adiacenti alle mura, si può dedurre che dai suoi inizi (X-XI secolo) esso è stato progettato come monastero, ma questo dato non esclude a priori il suo utilizzo come castello durante i combattimenti. Il materiale archeologico rinvenuto, datato tra la fine del XI secolo e l'inizio del XII secolo, non convalida la teoria di un suo utilizzo bellico. Sta di fatto che Apollonia in rapporto a Glavinica e Valona si trova distante e non poteva certo servire durante i combattimenti. Gurëzeza e Mashkjeza sono situate sul retro di Glavinica e molto vicino ad essa, hanno pertanto più probabilità di essere identificate con Mylus.

Secondo la scena indicata da Anna Comnena, sul suo cammino, Cantacuzeno giunse ad una piccola fortezza chiamata Il forte di Mylus e, subito, mise su i motori d'assedio e lo assediò. I romani si avvicinarono alle mura senza trovare ostacoli, alcuni gettavano il fuoco sulle porte e le bruciavano, mentre altri, si arrampicavano sulle mura e raggiungevano i bastioni. Direttamente i Franchi, che si erano accampati dall'altra parte del fiume Buses (Vjosa), se ne accorsero e corsero verso Mylus. Gli esploratori di Cantacuzeno - che erano barbari come ho spiegato - li videro, si misero a correre in maniera disordinata e non lo informarono in privato di quello che avevano visto ma cominciarono a gridare da lontano ed a raccontare l'avanzata dei Franchi. Quando i soldati sentirono parlare dell'approccio dei Franchi, anche se avevano scalato le mura e bruciato le porte, e stavano per catturare il forte, furono presi dal panico e ognuno corse a trovare il suo cavallo, ma tale era il loro terrore e la confusione mentale che saltarono l'uno sui cavalli dell'altro. Riordinati da Cantacuzeno, i soldati, bruciarono i motori d'assedio ed anche le barche sul fiume Buses per evitare che i Franchi trovassero un mezzo facile per attraversarlo ${ }^{29}$. Quando Cantacuzeno vide che anche lui (Exousiocrator Rosmices) era in difficoltà, prese coraggio come stimolato da qualcosa, si precipitò nella parte anteriore della linea dei Franchi e li inseguì fino alla fortezza di Mylus. Dopo aver ucciso molti degli ufficiali di secondo rango ed anche dei ranghi più alti e prendendo vivi alcuni conti illustri, come Ubus e suo fratello Riccardo e Contopaganus, tornò vittorioso ${ }^{30}$.

17 Un dato interessante, è il diploma di Michele II, despota dell'Epiro, scritto all'inizio dell'anno 1258, in occasione del matrimonio di sua figlia, Elena, con Manfredi, principe di Taranto "Michael II, Epiri despota, filiae suae Helenae Manfredo, principi Tarentino matrimonio coniungendae praeter insulam Corcyram et arces Subotum et Butrontum in dotem etiam castra quaedam et terras in Albania: Dyrrhachium, Avlonam, Caninam, Belgradum, montes Sfinarizae tradit ${ }^{31}$. "

18 Le proprietà che vengono date in dote mostrano anche un riconoscimento dei diritti del principe di Taranto in queste terre come eredi del regno normanno, che è anche sottolineato in un documento del 23 febbraio dello stesso anno in cui viene affermato :

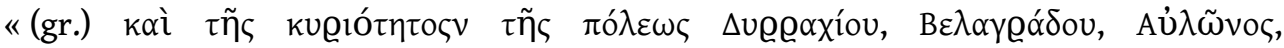

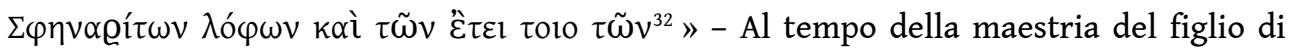


Federico II, Manfredi, che è sotto la benedizione del grande Signore, il principe di Taranto e il signore della montagna sacra $S$. Angelii (lat.), nel primo anno della sua proprietà possedeva Dyrrhachii, Belgrado, Avlonae, Montium Spinarizae e le province di queste aree.

\section{La ricerca archeologica}

19 Le ricerche archeologiche nell'area di Cakran iniziarono durante la prima guerra mondiale dagli storici G. Veith e C. Praschniker che videro le rovine di Floq e Gjonça e visitarono le rovine di Gurëzeza. Nel 1963, l'archeologo B. Dautaj continuò la sua ricerca scoprendo la stazione neolitica nella Valle di Cakran. Nel $1967 \mathrm{fu}$ condotta un'indagine di due settimane sulla stazione neolitica di Cakran e nel 1969-1970 gli scavi sistematici furono eseguiti da M. Korkuti e Zh. Andrea. Tra il 1976 e il 1981, N. Ceka e S. Muçaj effettuarono un gran numero di spedizioni di informazione nell'area. Nel 1983, N. Ceka, S. Muçaj e B. Vreka hanno scavato a Mashkjeza. Nello stesso anno, sulla collina di Gurëzeza, N. Ceka ha condotto indagini sul muro di cinta e sulla parete del terrazzo. Nel giugno 2006, L. Koço e O. Lafe hanno condotto ricerche superficiali nella città di Gurëzeza.

Per quanto riguarda la situazione dei siti scavati, la cresta collinare tra le due cime e il versante sud-occidentale insieme alla terrazza nella parte inferiore di essa è stata inclusa all'interno di una fortificazione rettangolare di $2100 \mathrm{mt}$. e una superficie di 23 ettari (cf. fig. 3/1). L'antico piano di Gurëzeza ha la forma di un quadrilatero irregolare completamente sostenuto sul pendio sud-occidentale della collina. Ci sono tre principali periodi di costruzione da distinguere. Il primo è legato ad una fortificazione medievale con un'area di circa 0,7 ettari ha che occupa solo la sommità della collina più alta (cf. fig. 3/3).

21 La fortificazione medievale circonda un'area di 0,7 ettari $^{33}$, rispetto ai 23 ettari di città antica $^{34}$. Il piano ellittico allungato segue la parete settentrionale dell'antica cinta muraria ${ }^{35}$, mentre sul lato est attende la parte posteriore formando un angolo retto rispetto alla parete nord. Sul bordo orientale c'è una torre quadrata con nervature di 5 mt. La parete meridionale segue il terreno piuttosto che il muro dell'antica acropoli, che utilizza come substrato solo nella sua parte occidentale. Questo lato ha anche una torre rotonda con un diametro di 8,6 mt. ed un'uscita di $5 \mathrm{mt}$. che Praschniker ha scambiato per una torre rotonda ${ }^{36}$. Sul lato ovest, la chiusura è ellittica e sembra che un tempo fosse protetta da una torre quadrilatera le cui tracce non sono chiare.

Gli scavi hanno rivelato diverse tecniche di costruzione. Nelle parti meglio conservate sul lato sud, la tecnica della costruzione delle pareti è chiaramente distinta. Sul sito e sul nucleo sono state collocate pietre di piccole e medie dimensioni e una grande quantità di mattoni ellenistici (dim. 0,38 x 0,39 x 0,08 mt.) riutilizzati (cf. fig. 3/4), leganti da un abbondante malta di calce e sabbia fine. Dalla tecnica di costruzione delle mura di cinta e dalle caratteristiche della planimetria, la città di Gurëzeza si trova più vicino a Bylis III e al castello di Margëlliç.

Diverse costruzioni sono state scoperte all'interno del recinto del castello, a partire dai resti di una chiesa. Nella parte centrale della piattaforma superiore della collina piatta, con un orientamento nord-ovest al punto più alto (quota $512.3 \mathrm{mt}$.), si trovano le rovine di una chiesa a singola navata - larghezza massima 8,30 mt., lunghezza $7 \mathrm{mt}$., spessore delle pareti della chiesa $0,65 \mathrm{mt}$; ; con le rovine di una torre quadrata posta al suo 
interno (est-ovest) 5,5 mt., larghezza (nord-sud) $6 \mathrm{mt}$., spessore della parete $1 \mathrm{mt}$. (fig. 4).

Fig. 4 - La chiesa e la torre di Gurëzeza

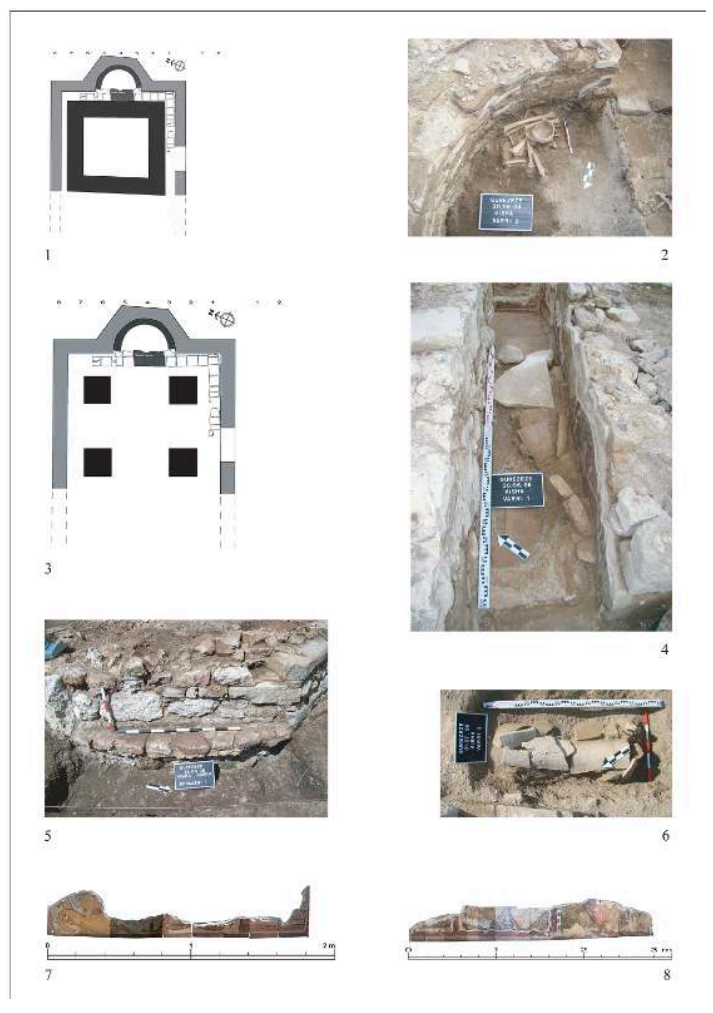

S. Bushi

Con l'espansione dei territori da loro occupati, i nobili Normanni, decidono di agire come nel caso dell'invasione del sud Italia e della Sicilia con la creazione di aree di sosta, con tutte le caratteristiche di una piccola città. Di solito per queste soste, il luogo più adatto doveva essere salubre, situato in altura - qui soddisfano le condizioni sia le costruzioni in Gurëzeza, così come quelli in Mashkjeza -, che era costruito, in un primo momento, probabilmente in legno - come la capanna con approvvigionamenti in Mashkjeza.

Il rilievo, ma anche le indagini condotte indicano che il castello occupava la parte centrale della piattaforma, ma l'aumento del numero di residenti ha provocato che l'abitato si è spostato al di fuori delle mura perimetrali della fortificazione, in una posizione più estesa (fig. 5). 
Fig. 5 - Gurëzeza. La residenza feudale
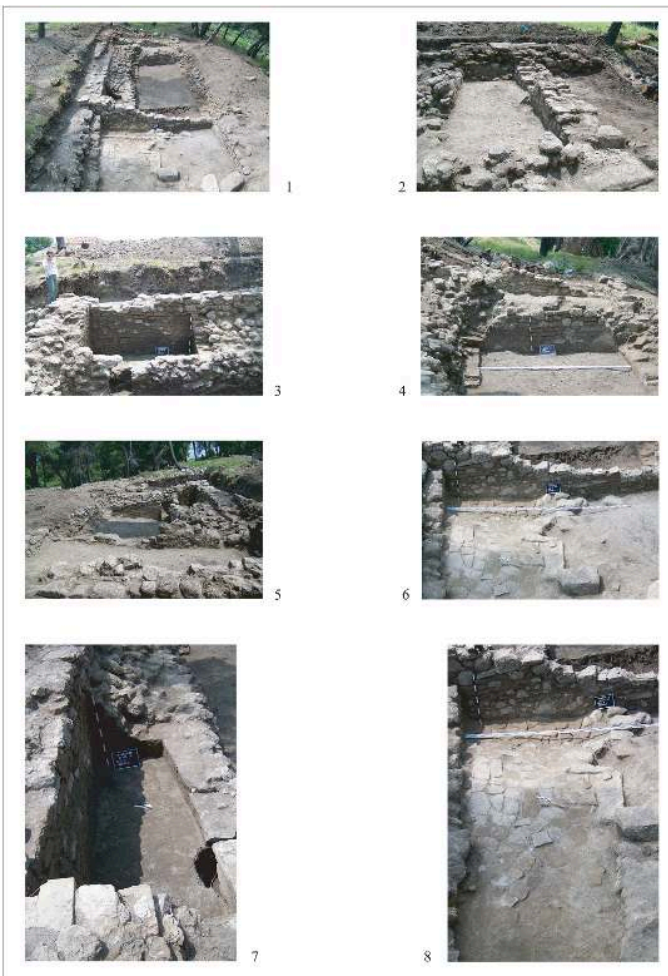

S. Bushi

Dato che le costruzioni del periodo antico di Gurëzeza erano ben conservate e sostituire o modificare richiedeva tempo e fatica, la costruzione del centro medievale fu spostata sull'acropoli che era vuota e sulla faccia opposta ove non esistevano grandi costruzioni del periodo del occupazione antica - locali scoperti sul lato nord, lunghezza complessiva 17,90 mt., larghezza complessiva 6,50 mt., lo spessore delle pareti varia da 0,70 a $1 \mathrm{mt}$.

Per quanto riguarda la cronologia, potremmo pensare che la costruzione della residenza sia iniziata dopo la costruzione della fortificazione e della chiesa. Gli edifici comprendono : ambienti residenziali (cf. fig. 5/1), aree per le riserve alimentari (cf. fig. 5/2), e una cisterna d'acqua (cf. fig. 5/3.5.7). I muri sono in pietra intrecciati con frammenti di mattoni disposti orizzontalmente e verticalmente (cf. fig. 5/4), secondo una tecnica nota nelle chiese della fine del'X secolo - inizio del XI secolo (cf. fig. 5/6.8), ma in questa costruzione, a differenza delle chiese, le mura sono state trovate costruite a secco (cf. fig. 5/2.8).

Gli scavi hanno anche permesso di osservare le strutture di una residenza feudale. Gli ambienti sono pavimentati con mattoni frammentati, mentre le soglie e telai delle porte sono di pietra (cf. fig. 5/6.8). Tale tecnica di costruzione e pavimentazione è stata riscontrata anche nelle strutture ausiliarie del monastero di Santa Maria, Apollonia, scoperto nel 2003. Il palazzo medievale è sostenuto da questa cortina muraria in pietra, posta sulla terrazza, un noto sistema del periodo medievale, con il quale si creava una sorta di fortificazione (cf. fig. 3/4).

In cima alla collina, nello stesso luogo in cui si trova la chiesa, si trova la torre o dongione, che è anche l'unico elemento protettivo in cui viene utilizzato il calcestruzzo 
(cf. fig. 4/1.4). Per la costruzione della torre è stata utilizzata la superficie interna della chiesa, per la costruzione dei suoi bordi sono stati utilizzati dei pilastri appiattiti mentre le fondamenta della torre sono state poste sul pavimento in mattoni della chiesa (cf. fig. 4/3). La torre ha dimensioni ridotte, forma quasi quadrata e pareti dello stesso spessore. Le pareti della torre sono fatte di pietra e piastrelle in pietra di medie dimensioni. Di solito, quella tipologia di torre, comprendeva diversi piani, uno dei quali era utilizzato anche per l'alloggio ${ }^{37}$.

Nella fila di ambienti scavati era inclusa anche una cisterna a forma rettangolare, di media grandezza, rivestita su tre lati con cocciopesto, tranne il lato rivolto verso il muro di cinta (cf. fig. 5/7). Questo era l'unico ambiente le cui pareti erano con calce bianca di alta qualità, a prima vista identica a quella usata per costruire la torre. La cisterna era montata su un muro con un sistema di scarico con tubi di ceramica l'argilla era la stessa utilizzata per le ceramiche da cucina. Non è noto se la cisterna fosse coperta, ma potremmo pensare che fosse coperta da un coperchio di legno.

Il materiale medioevale trovato appartiene all'ultimo periodo di vita della fortificazione, oltre ai tubi di ceramica per la raccolta dell'acqua nella cisterna - va detto che in entrambe le osservazioni, dei fondi dell'Istituto di Archeologia a Tirana e del Museo Storico di Valona, non hanno visto tubi con tale argilla, la stessa cottura e composizione del vasellame da cucina - in quantità maggiore sono i contenitori da cucina, utilizzati come contenitori di riserva (fig. 6 e 7) mentre in quantità limitata sono i contenitori in ceramica smaltata (fig. 8). Le ceramiche da cucina sono grandi pentole o padelle, senza maniglia (cf. fig. 6/1-2 e 7/1-8).

Fig. 6 - Gurëzeza. Ceramica da cucina

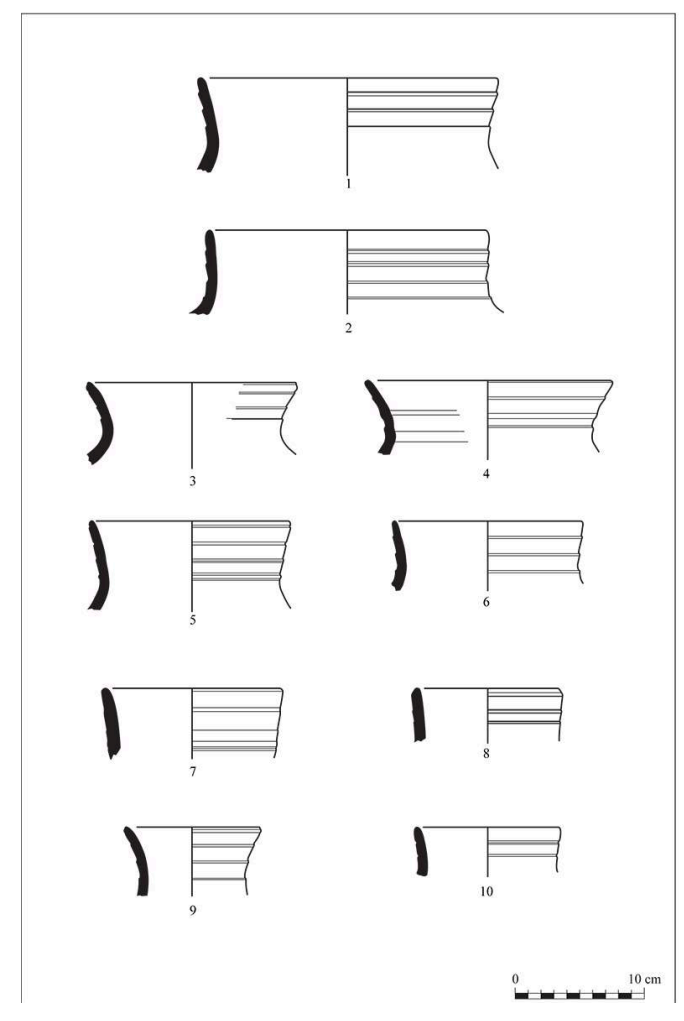

S. Bushi 
Fig. 7 - Gurëzeza. Ceramica da cucina

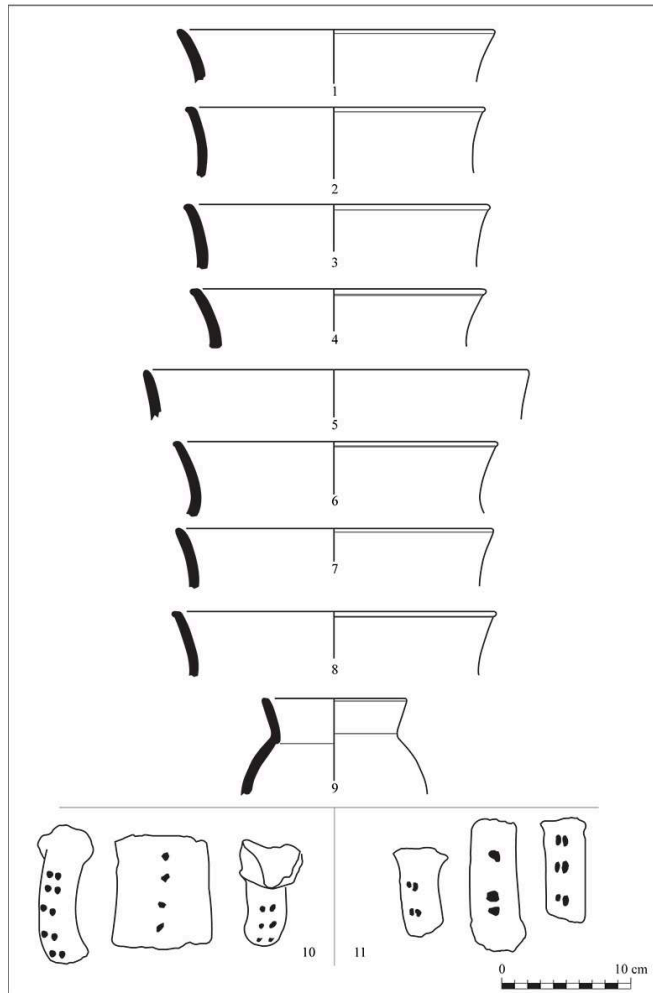

S. Bushi

Fig. 8 - Ceramica invetriata da Gurëzeza

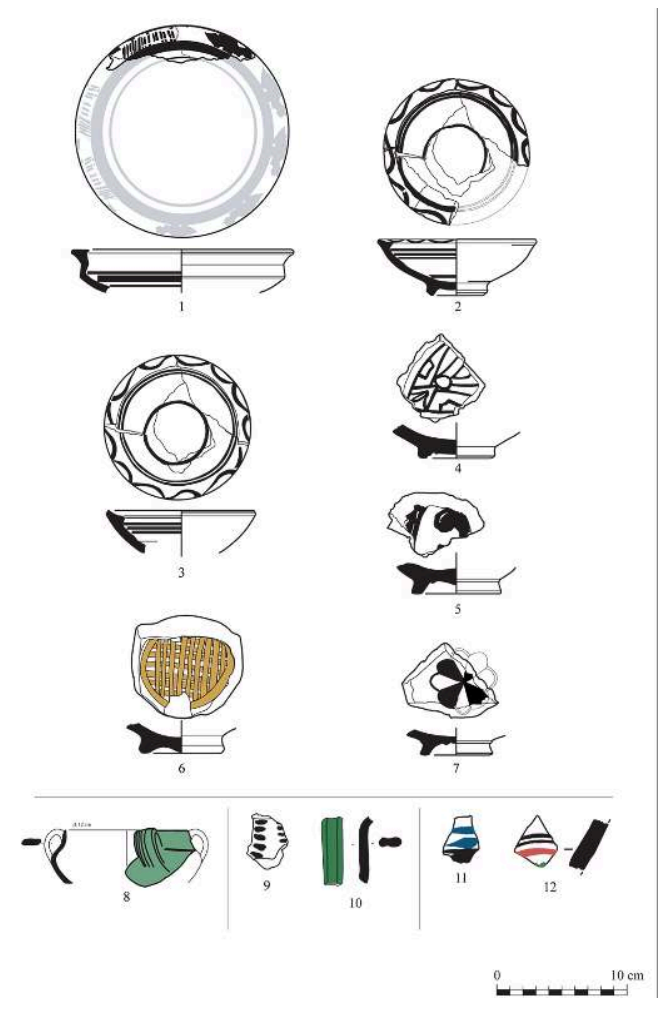

S. Bushi 
Le ceramiche smaltate sono del tipo protomaiolica in monocromia (cf. fig. 8/1-5.7.9), brindizine "grid iron» (cf. fig. 8/6.12), con smalto verde (cf. fig. 8/10) e Metallic Ware (cf. fig. 8/8) tutti datati alla fine del XIV secolo.

\section{Conclusione}

33 Alla luce della ricerca effettuata, è possibile collegare le strutture trovate a Gurëzeza ad altre simili situate nel Sud Italia. In effeti, sono stati trovati esempi di questi insediamenti, costruiti principalmente su siti abitati in periodi precedenti, che sono caratterizzati da una torre o dongion ${ }^{38}$, con pianta quadrata, di due o tre piani. Un muro perimetrale separava la residenza dalla zona contadina. Nel territorio d'Italia tutte queste fortificazioni furono costruite da Ruggiero I e Ruggiero I I ${ }^{39}$. Simile alla torre di Gurëzeza è quella di Paternò (6,3 mt.) e Adrano (4 mt.) in Sicilia, Scribla ${ }^{40} \mathrm{e}$ Satriano ${ }^{41}$, dove quest'ultimo come materiale e periodo di abbandono è lo stesso di Gurëzeza.

Le caratteristiche e la cronologia del materiale ceramico solleva la questione della fine dell'occupazione del sito fortificato di Gurëzeza. La posizione strategica di questa fortezza ne fa il centro di numerosi conflitti, almeno alla fine del XIV secolo. Dopo essere venuto al potere Federico II - dopo il suo ritorno dalle Crociate tra il 1230 e il 1250 -, questi centri stabiliti dai Normanni sarebbero stati posti direttamente sotto il potere imperiale. Con l'avvento al potere di Carlo d'Angiò, nel 1270, quando quest'ultimo era di ritorno da una crociata ${ }^{42}$, queste unità amministrative includevano il castello, un monastero e la popolazione. Ancora non sappiamo di eventi storici che si collegano alla fine di questa fortezza. L'unico dato certo è che le ceramiche risalgono alla fine del XIV secolo. Anche in città come Berat, ma anche vicino centro di Mashkjeza la ceramica da cucina è associata a questo tipo di ceramica smalta datata nello stesso periodo.

35 I frequenti cambiamenti nell'equilibrio est-ovest durante il XII secolo è ben espresso in molte aree come le costruzioni di difesa, gli edifici del culto cristiano, esportazione e importazione di merci, circolazione di monete, ecc. I castelli in questo territorio sono numerosi, si trovano sulle strade principali o in aree di importanza economica, per la maggior parte sono costruiti sulle rovine di castelli preistorici e antichi, compresa tutta o parte della superficie precedente. Finora in questo territorio siamo riusciti a scavare solo parzialmente nel centro di Gurëzeza e Mashkjeza. Da quelli che sono individuati ma non ancora scavati, evidenziamo il castello a Petran e Guri i Qytetit - it. Pietra della Città - a Përmet.

Reçu : 28 février 2018 - Accepté : 20 décembre 2018

\section{NOTE}

1. S. ANAMALI, K. BIÇOKU, F. DUKA, S. ISLAMI, M. KORKUTI, S. NAÇI, F. PRENDI, S. PULAHA e P. XHUfi, Historia e popullit shqiptar, t. 1, Tiranë, 2002, p. 225.

2. S. ANAMALI et alii, Historia..., ibid., p. 228. 
3. S. ANAMALI et alii, Historia..., ibid., p. 228.

4. S. ANAMALI et alii, Historia..., ibid., p. 228.

5. S. ANAMALI et alii, Historia..., ibid., p. 228. Vedi anche S. XHYHERI, Korridori Vlorë-Prespë, 1081-1385, Tezë Doktorature, Tiranë, 2016 [it. Il Corridoio Valona-Prespa, 1081-1385, Tesi di dottorato, Tirana, 2016].

6. S. ANAMALI et alii, Historia..., ibid., p. 228.

7. S. ANAMAli et alii, Historia..., ibid., p. 228.

8. Dello stesso anno sembra essere anche l'iscrizione sulla tomba di Roberto Monteforti scolpita nella stessa colonna dell'iscrizione del re bulgaro Boris, cf. C. PRASCHNIKER, Muzakhia und Malakastra, Wien, 1920, col. 196-198.

9. C. PRASCHNIKER, Muzakhia..., ibid., col. 100 e 196-198, fig. 112.

10. ORDERICUS VITALIS, Historia Æcclesiastica, lib. XI, 24, in M. CHIBNALL (ed.), The Ecclesiastical history of Orderic Vitalis, t. 6, Oxford, 1978, p. 101-105; vedi F. CHALANDON, Histoire de la domination normande en Italie et en Sicile, Paris, 1907.

11. C. PRASCHNIKER, Muzakhia..., op. cit., col. 197-198. Preschniker dice che, dopo l'assegnazione cronologica dalla riga 11 e oltre, la morte di Roberto, deve essere avvenuta il 20 settembre e forse l'anno è stato segnato con della vernice; per questo quando lui ha ispezionato la colonna non era visibile.

12. L.-R. MÉNAGER, «Inventaire des familles normandes et franques émigrées en Italie méridionale et en Sicile ( $\mathrm{xI}^{\mathrm{e}}-\mathrm{XII}{ }^{\mathrm{e}}$ siècles)», in Relazioni e comunicazioni nelle Prime Giornate Normanno-Sveve del Centro di studi normanno-svevi, Bari, 1973, Roberto il Guiscardo e il suo tempo, Roma, 1975, p. 260-390, ristampa in L.-R. MÉNAGER, Hommes et institutions de l'Italie normande, London, 1981, IV, p. 329.

13. Gesta Normannorum Ducum, lib. VII.17 (38), in The Gesta Normannorum Ducum of William of Jumièges, Orderic Vitalis, and Robert of Torigni, ed. E. M. C. VAN HOUTS, t. 2, Oxford, 1995, p. 176-177.

14. R.-N. SAUVAGE, Le Fonds de l'abbaye de Saint-Étienne de Caen aux archives du Calvados, t. 1, Caen, 1911, p. 271 ; per saperne di più vedi B. POHL, Eight Twelfth-Century Charters from the Norman Abbey of Saint-Étienne de Caen, c.1120-1204 (LoNDON, British Library, Add. Chs. 67574-67581) - A Catalogue and Commentary, Electronic British Library Journal (eBLJ), n 1, 2017, p. 1-52.

15. Recueil des actes de Henri II, roi d'Angleterre et duc de Normandie, concernant les provinces françaises et les affaires de France, éd. É. BERGER, t. 2, Paris, 1920, p. 375.

16. Materiale non ancora pubblicato. Le informazioni scambiate grazie l'autore della ricerca Dr. Elio Hobdari, Instituti i Arkeologjisë Tiranë.

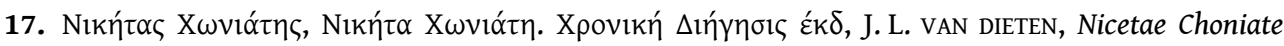
Historia, Berolini, 1975.

18. Il toponimo Gurëzeza, che fa parte ormai della letteratura archeologica, proviene da C. PRASCHNIKER, Muzakhia und Malakastra. Archäologische Untersuchungen in Mittelalbanien, Jahreshefte des Österreichischen Archäologischen Institutes in Wien, Band XXI-XXII, Wien, 1922-1924, p. 64-67. La collina è chiamata dai locali Pietre Nere a causa delle rocce bituminose che si trovano lì.

19. N. CEKA, «Fortifikimet antike të bashkësisë byline », Iliria, 20/1 (1990), p. 130-135, fig. 27.

20. Boemundo prese Petroula e la fortezza chiamata Mylus, che si trova dall'altra parte del fiume Deabolis, ed altre città simili alla periferia di Dyrrachium, che aveva ricevuto come parte della legge di guerra. Ha fatto questi atti con la forza militare; ma, allo stesso tempo, stava anche costruendo macchine da guerra (ANNA COMNENA, libro XIII, II, The Alexiad, tradotto da E. A. S. DAWES, Ontario, 2000, p. 232). La traduzione italiana del testo di Anna Comnena è stata fatta dall'autore di questo testo.

21. ANNA COMNENA, ibid., p. 238-239.

22. M. ŠUfFLAY, C. JIREČEK e L. THALlóczy, Acta et diplomata res Albaniae mediae aetatis illustrantia I, Vienna, 1913, nº 245.

23. M. šUfFlAY et alii, Acta et diplomata..., ibid., $\mathrm{n}^{\circ} 245$. 
24. Se dovessimo riconoscere che Cantacuzeno lasciò Këlcyra (Petra) - le rovine della città e un quartiere superiore di Këlcyra che è vicino alle rovine porta il nome di «Guri » (it. pietra) o dal castello di Petra (Petran, Përmet) per raggiungere Glavinica per seguire due sentieri, la più breve era la valle di Dishnica, Qafën e Kiçokut, Qafën e Gllavës, Rabie, Gjerbës, Aranitas, Ballsh - ; la più lunga, ma passando attraverso le pianure, avrebbe seguito la valle di Vjosa per andare a Drenova. 25. A. Ducellier afferma che Apollonia sia la più adatta : visto che il generale bizantino, prima di invadere Glavinica, intraprese l'attacco a Mylus per rompere il legame tra Valona e Durrazzo e per impedire ai Normanni di interferire con altre forze dal mare, cf. A. DUCELLIER, La façade maritime de l'Albanie au Moyen Age. Durrazzo et Valona du XI au XVe siècle, Thessalonique 1981, p. 24-25.

26. S. MUÇAJ, «Vendbanime të antikitetit të vonë në krahinën e Mallakastrës », Iliria, 9-10 (1979-1980), p. 277-299 ; s. MUÇAJ, «Kontribut për hartën arkeologjike të luginës së Gjanicës », Iliria, 7-8 (1977-1978), p. 331-337.

27. H. SPAHIU, « Monedha bizantine të shek. V-XIII të zbuluara në territorin e Shqipërisë », Iliria, 9-10 (1979-1980), p. 353-422, tav. I-VIII.

28. A. DUCELLIER, La façade maritime..., op. cit., p. 24-25.

29. ANNA KOMNENA, Libri XIII, V, p. 238. La traduzione di questo testo è stata fatta dall'autore.

30. ANNA KOMNENA, Libri XIII, VI, p. 239.

31. M. ŠUfFlAY et alii, Acta et diplomata..., op. cit., p. 71, $\mathrm{n}^{\circ} 245$ (traduzione di A. BUDA, I. ZAMPUTI, K. FRASHËRI e P. PEPO, Burime të zgjedhura për historinë e Shqipërisë (shek. VIII-XV), Tiranë, 1962, t. 2, p. 91, n 44): «Michele II despota d'Epiro, a la figlia Elena, che si unirebbe in matrimonio con Manfredi, il principe di Taranto, dà in dote a parte l'isola di Corfù, i castelli di Sopot (Subotum) e Butrint (Butrontum) alcuni castelli e luoghi di Arbëria: Durrazzo, Valona (Avlonam), Kanina (Caninam), Berat (Belgradum), e "rapporti/guadagni" nelle montagne di Spinarica (montes Sfinarizae). "

32. M. ŠUfFLAY et alii, Acta et diplomata..., ibid., p. 71, $\mathrm{n}^{\circ} 246$.

33. C. PRASCHNIKER, Muzakhia und Malakastra. Archäologische Untersuchungen..., op. cit., p. 65-67; S. MUÇAJ, « Vendbanime të antikitetit... », op. cit., p. 288-290.

34. N. CEKA, « Fortifikimet antike... », op. cit., p. 131.

35. C. PRASCHNIKER, Muzakhia und Malakastra. Archäologische Untersuchungen..., op. cit., p. 66, fig. 25 ; N. CEKA, « Fortifikimet antike... », ibid., p. 131-132.

36. C. PRASCHNIKER, Muzakhia und Malakastra. Archäologische Untersuchungen..., ibid., p. 66 e pianta.

37. F. CHIESA, «Les donjons normands d'Italie : une comparaison », Mélanges de l'École française de Rome - Moyen Âge, 110/1 (1998), p. 318.

38. F. CHIESA, « Les donjons normands... », ibid., p. 319 e 324.

39. F. CHIESA, « Les donjons normands... », ibid., p. 333.

40. A.-M. FLAMBARD-HERICHER, «Le chateau de Scribla. Une forteresse pour conquérir la Calabre », in Les Normands en Méditerranée aux XI ${ }^{e}$ et XII siècles, éd. Dossiers d'archéologie, 299 (2004-2005), p. 38-43.

41. D. Whitehouse, "Excavations at Satriano: a deserted medieval settlement in Basilicata", Papers of the British School at Rome, 38 (1970), p. 188-219.

42. Nel 1270 Carlo parti nell'ottava crociata, dove stipulò un nuovo trattato con il califfo Muhammad I al-Mustansir, nel quale il tributo veniva raddoppiato e Carlo otteneva anche il pagamento dell'intera indennità di guerra. Carlo poté poi rientrare in Sicilia nel novembre dello stesso anno, con mire ai territori dei Balcani. In A. KIESEWETTER, «L'acquisto e l'occupazione del litorale meridionale dell'Albania da parte di re Carlo I d'Angiò (1279-1283) », Palaver, 4 (2015), p. 255-298. 


\section{RIASSUNTI}

Lo scopo di questo articolo è di raccogliere prove storiche e archeologiche sulla presenza normanna in Albania. Infatti, nella primavera del 1081, un grande esercito normanno partì dal porto di Brindisi e Otranto, sotto la guida di Guiscardo e di suo figlio Boemundo. Quest'ultimo con parte della flotta sbarcò sulla costa di Valona, riuscendo, poi, a conquistare velocemente non solo la città stessa, ma anche dei luoghi strategici come Kanina, Jeriko (Orikum), Glavinica ecc. Il vero obbiettivo era la conquista della città principale sull'Adriatico, Durrazzo. Gli sforzi per la sua difesa risultarono inutili, e la città cade sotto i normanni. Dopo la conquista di Durrazzo, i normanni continuarono verso est, riuscendo a mettere sotto controllo l'ultimo territorio del « corridoio » Valona - Prespa, quello di Devoll. Dopo la morte di Guiscardo (1085), i normanni si ritirarono dall'odierna Albania, per fare ritorno nel 1107, guidati da Boemundo. La presenza dei Normanni è attestata a Glavinica, nel castello in Gurëzeza (Mylus) dove si trovano una chiesa ed i complessi residenziali, mentre la vicina Mashkjeza è una piccola unità militare, con una cisterna d'acqua, una cabina per le scorte alimentari e altri ambienti con funzione ancora indeterminata.

The purpose of this article is to gather historical and archaeological evidence on the Norman presence in Albania. Indeed, in the spring of 1081 a large Norman army landed on the coast of Vlora and within a short time, conquered the city and strategically important centers : Kanina, Jericho (Orikum), Glavinica, etc., and after they conquered Durrës, Norman armies took the last region the corridor of Vlora up to Prespa, passing through Devoll (Deabolis). After the death of Robert Guiskard (1085) the Normans withdrew from Albania, only to return again in 1107 under the direction of Boemund. After months of exhausting fighting, the Norman army, trapped in the Western Lowland (al. Ultësira Perëndimore), was forced to give up and return to Italy. The presence of the Normans in Glavinica is attested by the headstone inscription found in Ballsh dedicated knight Robert Montefort, which is identified with that of the XI-XII century family lived in Montfort-sur-Risle in Upper Normandy and the second son of Hugo II of Montfort. Of the same period is the castle in Gurëzeza (Mylus) where are located a church and the residential complexes, while in the nearby Mashkjeza is a small military unit, with a keep, a water cistern, a cabin for food stocks and several other rural environments yet undetermined function.

Le propos de cet article est de réunir les témoignages historiques et archéologiques sur la présence normande en Albanie. Au printemps de 1081, en effet, une grande armée normande débarqua sur la côte de Vlora et, en peu de temps, conquit la ville et les centres stratégiquement les plus importants : Kanina, Jéricho (Orikum), Glavinica, etc., jusqu'à Prespa, en passant par Devoll (Deabolis). Après la mort de Robert Guiscard (1085), les Normands se retirèrent d'Albanie pour ne revenir qu'en 1107 sous la direction de Bohémond. Après des mois de combats épuisants, l'armée normande, cernée dans les basses terres occidentales (soit l'Ultësira Perëndimore), est contrainte d'abandonner la contrée et de retourner en Italie. La présence des Normands à Glavinica est attestée par la pierre tombale du chevalier Robert de Montfort, second fils d'Hugues II de Monfort, de la famille seigneuriale de Montfort-sur-Risle en haute Normandie ( $\mathrm{XI}^{\mathrm{e}}$ $\mathrm{XII}^{\mathrm{e}}$ siècle). Le château de Gurëzeza (Mylus) appartient lui aussi à la période d'occupation normande. On y trouve une église et des complexes résidentiels. S'ajoute à Mashkjeza, à proximité, une petite unité militaire, avec une citerne d'eau, une cabane pour les réserves alimentaires et quelques autres installations rurales à la fonction encore indéterminée. 
INDICE

Mots-clés : Normands, tours médiévales, céramiques émaillées, XIe-XIVe siècle

Parole chiave : Normanni, Albania meridionale, torri medievali, ceramiche smaltate, sec. XI-XIV

Keywords : Normans, South Albania, medieval towers, glazed pottery, 11th-14th centuries

Indice geografico : Albanie du Sud

\section{AUTORI}

\section{SUELA XHYHERI}

Qendra e Studimeve Albanologike (Centre d'études albanologiques), Tirana, Albanie

\section{SKËNDER MUÇAJ}

Qendra e Studimeve Albanologike (Centre d'études albanologiques), Tirana, Albanie

\section{SKËNDER BUSHI}

Muzeu Historik Kombëtar (Musée historique national), Tirana, Albanie 